\title{
Activity-Centered Interaction Design: A Model-Driven Approach
}

\author{
Larry Constantine \\ Madeira Interactive Technologies Institute, \\ University of Madeira, Caminho da Penteada, \\ 9000-390 Funchal, Portugal \\ LConstantine@m-iti.org
}

Keywords: activity theory, activity modeling, model-driven design, interaction design.

\section{Extended Abstract}

Activity theory has had a long history and a growing influence in the design professions broadly conceived and in interaction design in particular. Leading authorities, including Donald Norman, Bonnie Nardi, and others, have called for and argued the advantages of design approaches that focus more on the activities in which human users engage than on the users themselves.

Human activity modeling is a recent development that builds on and enhances the effectiveness of activity theory by enabling more systematic and consistent modeling of core concepts in activity theory. Activity modeling makes possible a model-driven form of activity-centered design. Model-driven approaches are of growing influence in interaction design owing to the promise of yielding more orderly and manageable processes with enhanced traceability from initial conception and the establishment of requirements through to design and final realization.

The tasks that human users perform with software and other designed tools are always performed within the wider context of larger activities that need to be taken into account for the best interaction design. Human activities in the real world are invariably complicated by intricate and largely unpredictable interaction with other systems, artifacts, and other people. Activity theory and human activity modeling provide a simple, powerful framework for thinking about and making sense of how people use tools of all kinds and for setting the stage for delivering better, more usable solutions.

Through human activity modeling, activity-centered design becomes a more disciplined, predictable, and reproducible design process. Using a small set of simplified models and systematized descriptions, human activity modeling provides a concise format in which to capture, carry, and communicate the essence of user needs within human activities.

Human activity modeling provides a bridge between, on the one hand, the interests of interaction designers and other design professionals in understanding users and user needs and designing to support those needs more effectively, and, on the other hand, the interests of software engineers and software developers who most develop 
the products that actually function to support those needs. Human activity-modeling connects core constructs in activity theory to established concepts and techniques in software engineering, potentially improving communication between designers and developers and leading to a smoother overall development process.

This tutorial will introduce a systematic, activity-centered process for understanding user needs and for designing the interaction between users and software-based systems and services that better support those user needs. This approach grew out of and updates the highly successful and widely used practices of usage-centered design described in the award-winning book, Software for Use (Addison-Wesley, 1999). Activity-Centered Interaction Design is model-driven in the sense that simplified models guide and inform the full development process from concept to completed system. The focus is on understanding and modeling the activities in which users engage and then systematically deriving a design that directly and effectively supports users in performing those activities.

Presentations and discussions will be combined with hands-on small-group exercises and a case study problem will enable participants to experience and trace the entire model-driven design process through to a preliminary design.

Topics to be covered in this tutorial include:

- understanding human activity:

from activity theory to activity modeling

- human activity modeling: context models, participation models, and performance models

- understanding and modeling users within activities: user roles, personas, and role profiles

- activity-based task modeling: from activities to essential-form use cases

- model-based user interface architecture: navigation and content organization

- model-driven abstract prototyping: beyond wireframe schematics

- principle-driven interface design: model-driven derivation of design details

\section{References}

1. Constantine, L.L.: Human Activity Modeling: Toward a Pragmatic Integration of Activity Theory and Usage-Centered Design. In: Seffah, A., Vanderdonckt, J., Desmarais, M. (eds.) Human-Centered Software Engineering II. Springer, NY (2008)

2. Constantine, L.L.: Beyond User-Centered Design and User Experience. Cutter IT Journal 17(2) (February 2004)

3. Constantine, L.L., Windl, H.: Usage-Centered Design: Scalability and Integration with Software Engineering. In: Stephanidis, C., Jacko, J. (eds.) Proceedings of the 10th International Conference on Human-Computer Interaction: Theory and Practice, Crete, Greece. Lawrence Erlbaum Associates, Mahwah (2003)

4. Constantine, L.L., Lockwood, L.A.D.: Usage-Centered Engineering for Web Applications. IEEE Software 19(2), 42-50 (2002) 